\title{
Development of a Flood Warning Simulation System: A Case Study of 2007 Tewkesbury Flood
}

\author{
Chen Wang ${ }^{1, a}$, Yang Jiang ${ }^{2}$, Huan Xie ${ }^{3}$, David Miller ${ }^{1}$ and lain Brown ${ }^{4}$ \\ ${ }^{1}$ ICS, The James Hutton Institute, Aberdeen, UK, AB15 8QH \\ ${ }^{2}$ School of Computing Science and Digital Media, Robert Gordon University, UK, AB10 7QB \\ ${ }^{3}$ College of Surveying and Geo-Informatics, Tongji University, China, 200092. \\ ${ }^{4}$ Stockholm Environment Institute, University of York, UK, YO10 5DD
}

\begin{abstract}
Many flood warning systems were developed for 2D environments and limited on specific flood hazard. With the purpose of overcoming these disadvantages, it is necessary to propose new methodologies and techniques for 3D real time flood simulation. In this paper, a novel flood hazard warning system has been proposed. It describes and defines the relationship between the different parts of the simulation system in order to offer not only numeric data or figures, but also more meaningful and appealing 3D visual information. Consequently, the performance of this simulation system depends on the quality of the three sub systems: 3D real world modelling system with GIS data, 3D environment reconstruction system and 3D flood simulation system. A new flooding model has been developed which can handle dynamic flood behaviour and predict inundation areas in real time. In order to validate our flood warning system, the region of Tewkesbury in England has been simulated with a potential flood. The flood spreading process is shown during different time and the detailed inundation area is presented for further disaster evaluation. The study achieved two main objectives: implementing a useful flood simulation with real world model and reconstructed environment for flood hazard warning; producing a friendly simulation system interface for either a decision maker or experienced user.
\end{abstract}

\section{Introduction}

Simulating and modelling flood hazard are rapidly developing fields in hydrology[1]. Topographic data are crucial for flood inundation modelling and it is best to use recent and highly accurate topographic data. The flood simulation and model results are a good way of providing relevant information on how the flood is going to behave at the location where people live and how the flood will affect them. Current methods of flood hazard warning include numerical simulation[2][3][4][8], remote sensing approaches[5][6], rainfall data estimation[10] and GIS based flood simulation[7][9]. Although these methods can solve some piratical problems, there are still two disadvantages of the approaches: i) The flood prevention system is constructed in 2D environments other than 3D real world. ii) They forecast the flooding mainly based on the analysis of historical data.

There are increasingly requirements for developing efficient flood warning systems for decision making by government. Flood warning systems must be reliable and designed to operate during the most severe floods. In [11], a web-based flood forecasting system is presented to carry out real-time rainfall data conversion, modeldriven hydrologic forecasting, model calibration, precipitation forecasting, and flood analysis. However, the effectiveness of this system suffers from lack of flood spreading procedures. In [12], remotely sensed precipitation data and hydrologic model are used to monitor flooding in a region that regularly experiences extreme precipitation and flood events. This needs an offline process for data collection and also appears less efficiency for real time implementation. In[13], regional numerical weather prediction models are utilized, where high-resolution scale water balance and rainfall-runoff models are applied to output given basins, meteorological events and prediction products, and this approach is also found very weak to development of computationally tractable techniques to analyse how uncertainties cascade through a chain of linked non-linear models. In [14], a visualization approach for decision support is introduced by incorporating model uncertainty analysis, computationally efficient real-time data assimilation/forecasting algorithms, 2D inundation modelling, and data visualization. The efficiency needs more improvements for its capacity to introduce confusion by presenting information in an inconsistent fashion from one model or modelling approach to another. In [15], an investigation of the ability of highresolution TerraSAR-X synthetic aperture radar (SAR) data to detect flooded regions in urban areas is reported with a semiautomatic algorithm for the detection of

\footnotetext{
${ }^{a}$ Corresponding author: chen.wang@hutton.ac.uk
} 
floodwater in urban areas and its validation using aerial photographs. The main drawback of this approach is its poor display performance due to its $2 \mathrm{D}$ processing.

In this paper, the flood simulation of Tewkesbury has been conducted for validating the performances of our flood hazard warning system. This study achieved three main objectives: 1) implementing a useful flood simulation with real world model and reconstructed environment for flood hazard warning; 2) producing a friendly simulation system interface for either a decision maker or experienced user.

This paper is organized as follows: Section 1 gives an introduction of this simulation system architecture; Section 2 talks about the flowchart of the whole simulation system design and describes its components and their mutual relations; Section 3 presents a new dynamic terrain modelling and a new intelligent shape matching method for environment reconstruction; Section 4 describes a new flood simulation model for real time flood hazard warning and prevention; Section 5 reports experimental results to analyse its performances, and finally, Section 6 provides concluding remarks.

\section{The Simulation System Design}

This simulation platform is a centralized system. The architecture of the simulation platform is shown in Figure 1. As shown in figure 1 , the interface is in charge of three components of this project input and visualization output. The input includes the 3D real world modelling and user instruction, 3D environment reconstruction and 3D flood simulation. The output is mainly the simulation result. The 3D real world modelling is the basic step of the whole system that provides the terrain necessary for the simulation to be executed. At the beginning of simulation, the 3D real world enquires the elevation and image data via the interface and constructs the $3 \mathrm{D}$ real environments. During the modelling, 3D environment reconstruction system is added in for complicated and high resolution region reconstruction. Then, 3D flood simulation has been conducted based on the above two systems. It offers the flood region spreading algorithm with flood area contours to the simulation system and displays the simulation results via interface.

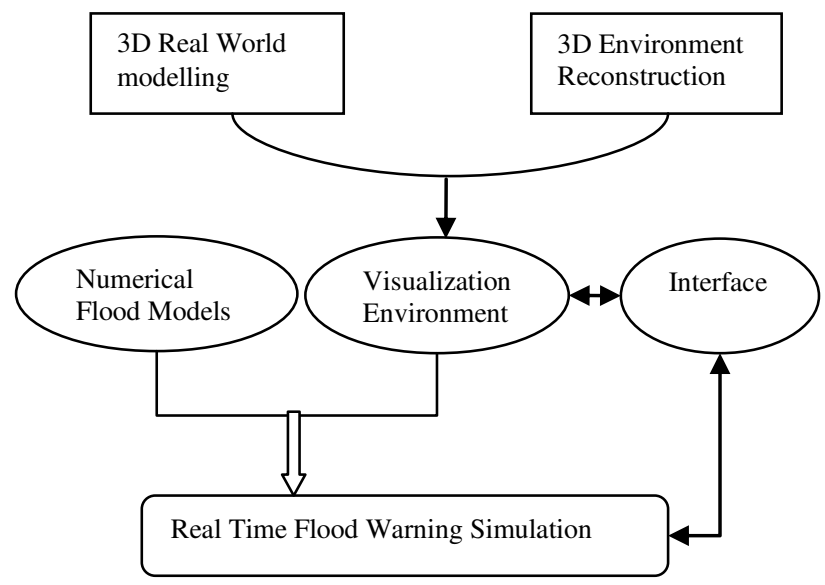

Figure 1. Framework for 3D visualisation and simulation of flooding
This simulation system is tested on a desktop computer under windows 7 operating system, the CPU is Intel(R) Core(TM) i7-3770 CPU, RAM 16GB and the video card is NVIDIA Quadro K2000 with 2GB GPU Memory.

A number of simulation experiments have been designed to test this simulation platform, which will be discussed in the following sections.

\section{3D Environment Modelling and Reconstruction}

Generating 3D real world is one of the major concerns while modelling the simulation environments. While constructing the 3D real world, firstly, the data of the environments is loaded from the external files, which include the terrain information and the image information; secondly the high level hierarchical terrain information is interpolated to form the detail elevation grid structure; at the end all the environmental information is combined together and the created terrain environments are displayed on the monitor screen. Although the real world model has been constructed, the details of the environment like vegetation or buildings are still not enough for viewers. It is necessary to recreate the whole models based on environment reconstruction system. In [17], we have proposed a novel approach regarding modelling and automatic reconstructing $3 \mathrm{D}$ virtual Environments. The experiment results show that our method is promising, and able to deal with massive GIS terrain data, automatically segment targeted image areas and rebuild environmental objects efficiently. The method has been applied to use in Tewkesbury 3D environment reconstruction and will carry on further flood simulation as the basic step.

\section{Real Time 3D Flood Simulation}

As the key component of 3D flood simulation, flood spreading model is proposed to move the flood front edges outwards, starting from their initial location that is the river boundaries. The flood model is integrated with the digital terrain by using the geometrical elevation data of the terrain or terrain profile map and the flood flow inundatiuon area can be deterimined with the given flood volume known. The proposed model is able to incorporate the flow rate of water volume of flooding, developed based on the method of C-V model [18].

Let $\mathrm{I}$ be the profile map of a flooding region, and $\mathrm{g}$ be edge function obtained from the map, and $\Omega$ be a terrain map domain, we define that $C(x, y)$ is a final flooded region boundary, and $\left\{\varphi_{0}=\varphi_{0}\left(\chi_{0}, y_{0}\right) ; \varphi(x, y) \in \Omega\right\}$ is the initial set of the moving boundary $\varphi(x, y) \in \Omega$. Thus we have

$$
g=1 /\left(1+\left|\nabla_{G_{\sigma}}(x, y) * I(x, y)\right|^{2}\right)
$$

where $G_{\sigma}(x, y)$ is a Gaussian function with zero mean and standard deviation $\sigma$, and * indicates a 
convolution operator. The flooding boundary energy can be defined by

$$
\begin{aligned}
& E_{\varphi}=E_{\text {boundry }}+E_{\text {area }}+E_{\text {inside }}+E_{\text {outside }} \\
& E_{\text {boundry }}=\lambda_{2} \int_{\Omega} g \cdot \delta_{\varphi}(x, y)\left|\nabla_{\varphi}(x, y)\right| d x d y \\
& E_{\text {area }}=\lambda_{1} \int_{\Omega} g \cdot H(\varphi(x, y)) d x d y \\
& E_{\text {inside }}=\lambda_{3} \int_{\Omega} H(\varphi(x, y))\left|u(x, y)-Z_{1}\right|^{2} d x d y \\
& E_{\text {outside }}=\lambda_{4} \int_{\Omega}\left(1-H(\varphi(x, y))\left|u(x, y)-Z_{2}\right|^{2} d x d y\right.
\end{aligned}
$$

where $\lambda_{1} \lambda_{2} \lambda_{3}$ and $\lambda_{4}$ are weighting constants, $H$ is the Heaviside function, $\delta$ is the Dirac delta function.

$$
\begin{aligned}
& H(\varphi)=\left\{\begin{array}{l}
1 ; i f \varphi \geq 0 \\
0 ; i f \varphi<0
\end{array}\right. \\
& \delta(\varphi)=\frac{d}{d \varphi} H(\varphi)
\end{aligned}
$$

The first two terms of Equation 2 are the energies related to the flooded region boundary and the flooded region itself respectively. The third and fourth terms of Equation 2 are global fitting energies contributed from the region inside the flood boundary and outside the flood boundary respectively. Functions $\mathrm{Z}_{1}$ and $\mathrm{Z}_{2}$ are weighted averages of the $\mathrm{z}$ values (elevation data) of the terrain inside and outside the flooded region boundaries.

The flood energies defined in Equation 2 drive the flooding boundary outwards during its evolution, until reaching a position, where the flood rate of water volume is balanced by a flood level held in the flooded region.

Assume that $Q_{g}$ is a known flow rate of flood volume at a time $\mathrm{t}$, and $Q_{i}$ is the flow rate of flood volume estimated during the evolution at each iteration step, using Euler-Lagrange partial differential equation, we have the flooding motion equation deduced from Equation (9) :

$$
\frac{d \varphi}{d t}=\delta(\varphi)\left(Q_{g}-Q_{i}\right)\left(\lambda_{1} \operatorname{div}\left(g \frac{\nabla_{\varphi}}{\left|\nabla_{\varphi}\right|}\right)-\lambda_{2}-\lambda_{3}\left(u-Z_{1}\right)^{2}+\lambda_{4}\left(u-Z_{2}\right)^{2}\right)
$$

\section{Case Study of Tewkesbury Flood}

Tewkesbury is a town of Gloucestershire in England. It stands at the confluence of the River Severn and the River Avon, and also minor tributaries the Swilgate and Carrant Brook. The area around Tewkesbury is frequently affected by flooding. In general such flooding causes little damage to property as the town is surrounded by large areas of floodplain which restrict urban development and the ability for the town to spread. However, extreme flooding events have caused damage to property and affected transport links, the most significant events occurring in 1947, 1960 and 2007.

\subsection{Description of Study Area}

In July 2007, River Severn and the River Avon which meet at Tewkesbury were overwhelmed by the volume of rain that fell in the surrounding areas on one day, Friday 20th July. Tewkesbury was completely cut off with no road access, parts of the town were under around 3 feet $(0.9 \mathrm{~m})$ of water and flood waters entered Tewkesbury Abbey for the first time in 247 years [16]. Tewkesbury's Mythe Water Treatment Works were flooded, threatening drinking water supplies to 350,000 people, and Severn Trent Water warned that treated water would run out by early Sunday evening in Tewkesbury. Combined military and civil emergency services tried to stop floods reaching the Walhamelectricity substation in Gloucester supplying half a million people.

\subsection{Available Data}

The case of flooding is in the upper part of the River Severn. The grid for the analyzed area was generated by using 3D real world modelling. We prepared this topographic data from the SRTM and converted the data into the DEM, whose coordinates from N52 $21^{\prime} 45^{\prime \prime}$ to $\mathrm{N} 52^{\circ} 21^{\prime} 58^{\prime \prime}$ and $\mathrm{W} 2^{\circ} 18^{\prime} 35^{\prime \prime}$ to $\mathrm{W} 2^{\circ} 18^{\prime}$ with $512^{*} 256$ nodes .The texture map for the surface of Tewkesbury terrain is obtained from Google Earth. The analyzed domain is $650 \mathrm{~m} * 325 \mathrm{~m}$. The forcing condition is a discharge of $820 \mathrm{~m}^{3} / \mathrm{s}$, which applied suddenly by a flood wave that moves down the centre of the city. The time step is 25 seconds and the total duration of simulation is 650 seconds.

\subsection{Texture Map Analysis and Segmentation}

The texture map of local region of Tewkesbury is obtained from Google Earth in Figure 2 (a). Before modelling this 3D terrain, image analysis such as specific areas segmentation is important. By using our active contours method, the whole environmental image has been segmented and identified with several distinct colours. Figure 2 (b) shows the processed image with different recognised specific areas: pink indicates the street area, yellow displays the houses, blue shows the river area, cyan represents garden areas, green is for forest area and light green is for grassland area.

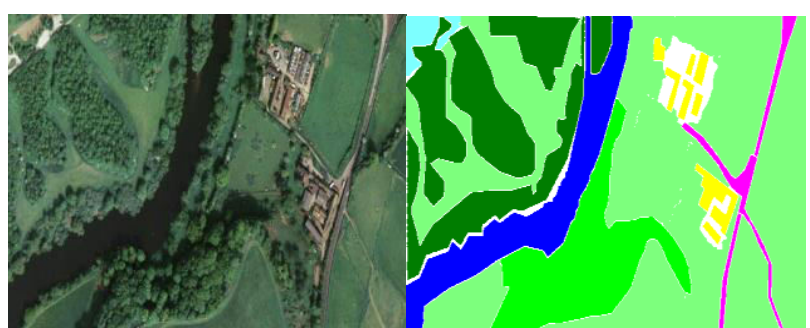

Figure 2 (a) and (b). Texture map and segmented image of local region of Tewkesbury. 


\subsection{Digital Terrain Modelling and Reconstruction}

For modelling the 3D environment of local region of Tewkesbury, we use the proposed dynamic terrain generation method which is mentioned in section 3 . SRTM data is used for generating 3D geometry of Tewkesbury and the satellite image is obtained from Google Earth. The generated terrain geometry is overlaid by satellite image corresponding to the same geographic area which is presented in Figure 3 (a). For terrain reconstruction, at first, we need to segment the image and select the objective areas for further study. Figure 3 (b) shows the local region of Tewkesbury reconstructed environment which is a nice platform for carrying out flood simulation.

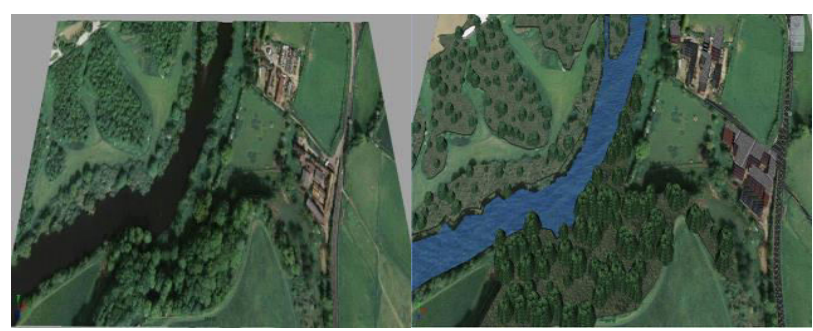

Figure 3 (a) and (b). 3D environment and reconstructed virtual model of local region of Tewkesbury

\subsection{Simulation Results}

We have computed flood flow direction and inundation areas at different stages by using the flood spreading model. This flood model based on standard gradient descent method for energy minimization can predict the flood inundation areas precisely. An easy to use interface for this flood simulation is designed and presented with several functions in Figure 4.

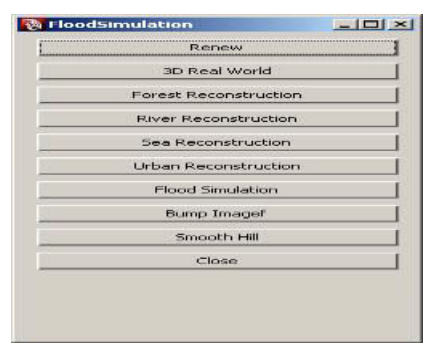

Figure 4. Interface of flood simulation system

When the river water starts overflowing its bank, flood hazard happens. The computed water elevation surfaces at $\mathrm{t}=75 \mathrm{~s}, \mathrm{t}=150 \mathrm{~s}, \mathrm{t}=250 \mathrm{~s}, \mathrm{t}=450 \mathrm{~s}$ and $\mathrm{t}=650 \mathrm{~s}$ are presented in the Figure 5.

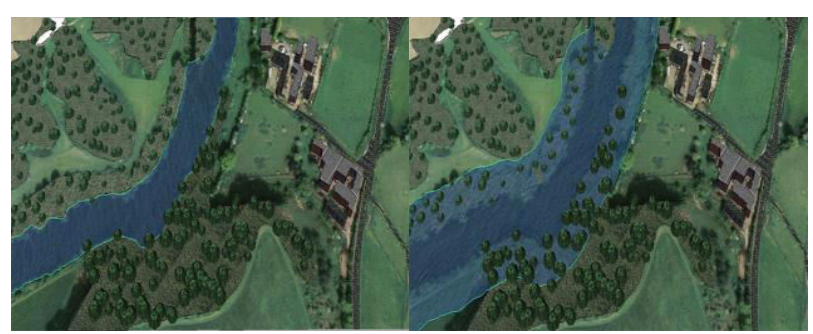

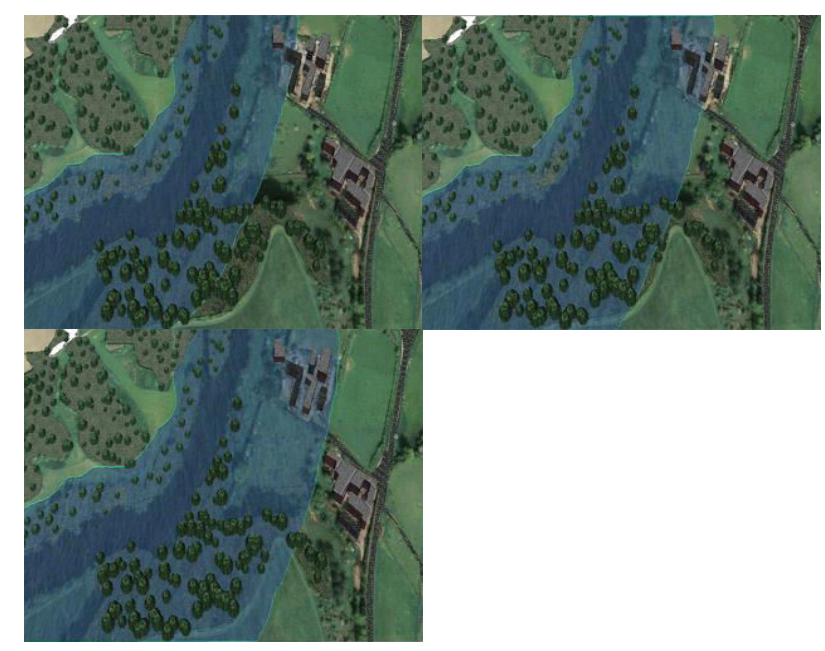

Figure 5. Local region of Tewkesbury flood spreading at different stages

\subsection{Discussion}

In this flood simulation test, satisfied experiment result has been achieved. This test is based on the 3D environmental reconstruction system which consists of environmental image segmentation for object identification, a shape match method and an intelligent reconstruction system discussed in previous sections. The active contours-based method is used for extracting necessary objects from images. The flood spreading model is useful for calculating the flood inundation area. The studies will eventually be used to determine the appropriate level of protection needed for Tewkesbury for future flood warning.

\section{Conclusion}

This paper discusses the simulation system architecture which includes 3D real world modelling, 3D environment reconstruction and 3D flood simulation systems. 3D real world modelling is composed of terrain and image data loading, the ROAM algorithm for single terrain modelling and the NineTreeNodes algorithm for dynamic terrain modelling. 3D environment reconstruction is made up of object segmentation based on active contours and intelligent shape match reconstruction. 3D flood simulation system consists of flood region spreading algorithm and simulating flood inundation by flood area contours. Our simulation platform offers convenient methods for constructing the real urban environments, specifying the flood hazard prevention tasks and monitoring the inundation areas.

\section{References}

[1] Boughton. W and Droop. O, Continuous simulation for design flood estimation--a review.Environmental Modelling \& Software, 18(4): 309-318.2003.

[2] Mark. O, Weesakul. S, Apirumanekul. C, Aroonnet. S.B and Djordjevic. S, Potential and limitations of 
1D modelling of urban flooding. Journal of Hydrology, 284-299, 2004.

[3] E. Mignot, A. Paquier and S. Haider, "Modeling floods in a dense urban area using 2D shallow water equations", Journal of Hydrology, 327 (1-2), pp. 186-199, 2006.

[4] Marks K and Bates P, Integration of high-resolution topographic data with floodplain flow models. Hydrol Process 14: 2109-22,2000.

[5] Wickland. D. E, "Future Directions for Remote Sensing in Terrestrial Ecological Research", In: Asar, G. (Ed.) Theory and Applications of Optical Remote Sensing. John Wiley and Sons Ltd., Chichester, pp. 691-72, 1989.

[6] Curran.P. J, Foody. G. M and Van Gardingen P. R. Scaling-up. In: van Gardingen, P. R., Foody, G. M. and Curran, P. J (Eds.) Scaling-up: From Cell to Landscape, Cambridge University Press. pp. 1-5, 1997.

[7] Liu Yaolin and Miao Zuohua, "Key Technique of Floods Prevention Information System Based on GIS" Geoscience and Remote Sensing Symposium, IGARSS'05, 2005 IEEE International Volume 6, 2529, Page(s):4395-4398, 2005.

[8] England JF Jr, Velleux ML, Julien PY. 2007, “Twodimensional simulations of extreme floods on a large watershed", Journal of Hydrology 347(1-2): 229241, DOI: 10·1016/j.jhydrol. 09.034, 2007.

[9] Jian Chen, Arleen A. Hill, Lensyl D. Urbano, "A GIS-based model for urban flood inundation", Journal of Hydrology, 373 (2009) 184-192, 2009.

[10] Alison L. Kaya, Nicholas S. Reynard and Richard G. Jones, "RCM rainfall for UK flood frequency estimation. I. Method and validation", Journal of Hydrology, Elsevier, 318 (2006) 151-162, 2006.

[11]Xiang Yang Li, K.W. Chaub, Chun-Tian Cheng, Y.S. Li, "A Web-based flood forecasting system for Shuangpai region", Advances in Engineering Software, Elsevier, 37 (2006) 146-158,2006.

[12] Kwabena O. Asante, Rodrigues D. Macuacua, Guleid A. Artan, Ronald W. Lietzow, and James P. Verdin, "Developing a Flood Monitoring System From Remotely Sensed Data for the Limpopo Basin", IEEE TRANSACTIONS ON GEOSCIENCE AND REMOTE SENSING, VOL. 45, NO. 6, JUNE 2007.

[13] AD P.J. DE ROO, BEN GOUWELEEUW, JUTTA THIELEN et al, "Development of a European flood forecasting system", Intl. J. River Basin Management Vol. 1, No. 1 (2003), pp. 49-59, 2003.

[14]D. Leedal, J. Neal, K. Beven, P. Young, P. Bates, "Visualization approaches for communicating realtime flood forecasting level and inundation information", Journal of Flood Risk Management Volume 3 Issue 2 pp 140-150,2010.

[15] David C. Mason, Rainer Speck, Bernard Devereux et al, "Flood Detection in Urban Areas Using TerraSAR-X", IEEE TRANSACTIONS ON GEOSCIENCE AND REMOTE SENSING, VOL. 48, NO. 2, FEBRUARY 2010

[16]FASW,http://www.gloucestershire.gov.uk/index.cfm ? articleid $=3328$.
[17] Wang. C, Wan T. R and Palmer I.J, "Automatic Reconstruction of 3D Environment Using Real Terrain Data and Satellite Images", International Journal of Intelligent Automation and Soft Computing, Vol 18, No. 1, pp.49-63, 2012

[18] Chan, T.F. and Vese, L.A.,. "Active Contours without Edges", IEEE Transactions on image processing, Vol.10, No. 2, pp.266-277, February (2001). 